\title{
Implementation and Comparison of Controllers for Planar Robots
}

\author{
John Kern*, Claudio Urrea ${ }^{\dagger}$ and Hugo Torres**
}

\begin{abstract}
The nonlinear behavior and the high performance requirement are the main problems that appear in the design of manipulator robots and their controllers. For that reason, the simulation, realtime execution and comparison of the performance of controllers applied to a robot with three degrees of freedom are presented. Five controllers are prepared to test the robot's dynamic model: predictive; hyperbolic sine-cosine; sliding mode; hybrid composed of a predictive + hyperbolic sine-cosine controller; and adaptive controller. A redundant robot, a communication and signal conditioning interface, and a simulator are developed by means of the MatLab/Simulink software, which allows analyzing the dynamic performance of the robot and of the designed controllers. The manipulator robot is made to follow a test trajectory which, thanks to the proposed controllers, it can do. The results of the performance of this manipulator and of its controllers, for each of the three joints, are compared by means of RMS indices, considering joint errors according to the imposed trajectory and to the controller used.
\end{abstract}

Keywords: Predictive control, Adaptive control, Hyperbolic sine-cosine control, Sliding mode control, Manipulator robot

\section{Introduction}

Currently robotics plays a very important role in industrial automation and in a country's development. In particular, the benefits of using robots in production lines are many, making it imperative to train future professionals who will work in this area.

El linear prediction model was initially represented by the response to impulse model called Model Algorithmic Control (MAC). Later it was shown that the conventional model approach MPC can be interpreted as continuous time state space model [1].

The Model Predictive Control (MPC) has been introduced mainly to tackle processes with complex dynamics and is now one of the most widely used advanced control methods in the process control industry $[2,3]$.

The term predictive controller does not design a particular control strategy but a set of control methods that make explicit use of a model of the process to get the control signal minimizing an objective function $[2,4]$.

The predictive controller has the purpose of solving efficiently control and automation problems of industrial processes that are characterized by presenting a complicated, multivariable, and/or unstable dynamic behaviour. The control strategy on which this kind of control is based uses the mathematical model of the process to be controlled to predict the future behaviour of that system, and based on

$\dagger$ Corresponding Author: Automation Group, Department of Electrical Engineering, University of Santiago of Chile, Chile.

(claudio.urrea@usach.cl)

Automation Group, Department of Electrical Engineering, University of Santiago of Chile, Chile.

School of Electronics, University of Azuay, Cuenca, Ecuador

Received: November 11, 2015; Accepted: November 29, 2016 that future behaviour it can predict the future control signal $[5,6]$.

It should be mentioned that aside from the classical PID controller, the MPC strategy has been widely accepted in the academic world and in industry [7].

The nonlinear behaviour and the high performance requirement are the main problems that appear in the design of manipulator robots and their controllers. For that reason, the objective of this paper is to design, simulate and compare the performance of controllers applied to a robot with three Degrees of Freedom (DOF). Five controllers are prepared to test the robot's dynamic model: predictive; hyperbolic sine-cosine; sliding mode; hybrid composed of a predictive + hyperbolic sine-cosine controller; and adaptive controller. A simulator is developed by means of the MatLab/ Simulink software that allows the dynamic performance of the robot and of the designed controllers to be analysed. The manipulator robot is made to follow a test trajectory which, thanks to the proposed controllers, it could follow. The results of the performance of this manipulator and of its controllers for each of the three joints are compared by means of RMS indices, for which purpose the joint errors in terms of the imposed trajectory as well as according to the controller used are considered.

\section{Manipulator Robot Control}

Control of the motion of a manipulator robot's joints is a current issue whose study is of great importance in order to make its terminal effectors follow a predefined trajectory with minimum deviations [8]. 
One application of the Model Predictive Control in a flexible link manipulator is aimed at solving the suppression of vibrations, which is a recurrent problem in most robotic applications. The solution of this problem will allow to increase the operating speed as well as the precision of the manipulators [9]. For example, in [10] artificial neural networks-based fault detection and isolation applications for robotic manipulators are presented. In [11] a predictive controller based on interpolation is applied, whose inputs are subject to some ties thanks to which a manipulator robot with two joints can be controlled. The predictive controller is inserted in an adaptive perturbation scheme and it corrects the commands to be applied to each of the joints, calculated by inverse dynamics, when the system is affected by an additive perturbation. The results obtained by means of computer simulations are compared with those obtained with other control strategies, showing the advantages of making the interpolation between different kinds of solutions to get a feasible solution, i.e., one that does not violate the system's ties.

Some new strategies have been used in recent years to control robots, but normally industrial robots still use a classical control of the PID type [12], even though their manipulators composed of several joints connected together have a highly nonlinear dynamic with strong coupling between their corresponding joints. This complicates the task of a simple PID controller as well as its tuning, particularly with high speed order consignments or accelerations. Better solutions have been proposed with controllers based on the robot's model, which use a mathematical model of the mechanism with the purpose of compensating for the existing dynamic terms [12].

The nonlinear behavior and the requirement of high performance are the main problems that appear in the design of manipulator robots and their controllers. For that reason efficiency and effectiveness are required in the control strategies for those manipulator robots to be able to fulfil a given task precisely. Another problem that appears in controller design is to determine a function that commands the actuators in such a way that the position of the effector terminal achieves a constant position trajectory in the face of external perturbations of the robotized system. Keeping in mind the difficulties that there are to solve the problem of control of manipulator robots, it is necessary to design, evaluate and compare different control strategies in order to find an adequate controller that can adapt to the specific operational needs of those robots.

\section{Kinematic and Dynamic Model for the 3-DOF Manipulator}

In order to evaluate the performance of various controllers when following the desired trajectories, a kinematic and a dynamic model are now described for the 3-DOF manipulator that is being studied.

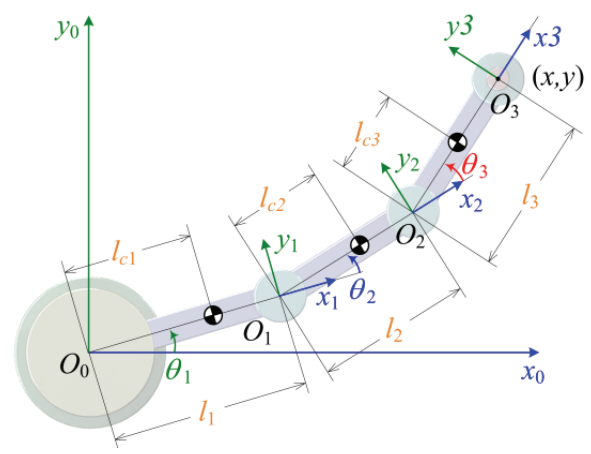

Fig. 1. Graphic representation of the 3-DOF manipulator on the $x-y$ plane

\subsection{Direct kinematics}

Considering the standard Denavit-Hartenberg algorithm, Fig. 1 describes the position and orientation of this manipulator on the $x-y$ plane with respect to its dextrogyrous orthogonal base system $\left(x_{0}, y_{0}, z_{0}\right)$.

Carrying out different homogeneous transformations the direct kinematics shown in Eq. (1) is obtained [16, 17]:

$$
{ }^{0} \mathbf{T}_{3}=\left[\begin{array}{cccc}
c_{123} & s_{123} & 0 & l_{1} c_{1}+l_{2} c_{12}+l_{3} c_{123} \\
s_{123} & -c_{123} & 0 & l_{1} s_{1}+l_{2} s_{12}+l_{3} s_{123} \\
0 & 0 & -1 & l_{0} \\
0 & 0 & 0 & 1
\end{array}\right]
$$

where,

$$
\begin{aligned}
& c_{1}=\cos \left(\theta_{1}\right) ; c_{12}=\cos \left(\theta_{1}+\theta_{2}\right) ; c_{123}=\cos \left(\theta_{1}+\theta_{2}+\theta_{3}\right) \\
& s_{1}=\sin \left(\theta_{1}\right) ; \mathrm{s}_{12}=\sin \left(\theta_{1}+\theta_{2}\right) ; c_{123}=\cos \left(\theta_{1}+\theta_{2}+\theta_{3}\right)
\end{aligned}
$$

\subsection{Dynamics}

For this purpose, we will employ the Lagrange-Euler formulation that is based on the principle of energy conservation. In this way, the dynamic model of a manipulator can be expressed by (2) [16].

$$
\boldsymbol{\tau}=\mathbf{M}(q) \ddot{\mathbf{q}}+\mathbf{C}(q, \dot{q})+\mathbf{G}(q)+\mathbf{F}(\dot{q})
$$

where, $\tau$ : Vector of generalized forces (forces and torques), M: Inertia matrix, C: Vector of centrifugal and Coriolis forces, G: Vector of gravitational force, $\mathbf{q}$ : Vector of generalized coordinates (joint), $\dot{\mathbf{q}}$ : Vector of generalized speeds (joint), $\ddot{\mathbf{q}}$ : Vector of joint acceleration, and $\mathbf{F}$ : Vector of friction forces. The dynamic model of the robotic manipulator can be expressed through Eqs. (3-14) [16, 17].

$$
\mathbf{M}=\left[\begin{array}{lll}
M_{11} & M_{12} & M_{13} \\
M_{21} & M_{22} & M_{23} \\
M_{31} & M_{32} & M_{33}
\end{array}\right]
$$




$$
\begin{gathered}
M_{11}=I_{1 \mathrm{zz}}+I_{2 \mathrm{zz}}+I_{3 \mathrm{zz}}+m_{1} l_{\mathrm{c} 1}^{2}+m_{2}\left(l_{1}^{2}+l_{\mathrm{c} 2}^{2}+2 l_{1} l_{\mathrm{c} 2} \mathrm{c}_{2}\right)+\ldots \\
m_{3}\left(l_{1}^{2}+l_{2}^{2}+l_{\mathrm{c} 3}^{2}+2 l_{1} l_{2} \mathrm{c}_{2}\right)+m_{3}\left(2 l_{2} l_{\mathrm{c} 3} \mathrm{c}_{3}+2 l_{1} l_{\mathrm{c} 3} \mathrm{c}_{23}\right) \\
M_{21}=M_{12}=I_{2 z z}+I_{3 z z}+m_{2}\left(l_{\mathrm{c} 2}^{2}+l_{1} l_{\mathrm{c} 2} \mathrm{c}_{2}\right)+\ldots \\
m_{3}\left(l_{2}^{2}+l_{\mathrm{c} 3}^{2}+l_{1} l_{2} \mathrm{c}_{2}+2 l_{2} l_{\mathrm{c} 3} \mathrm{c}_{3}\right)+m_{3} l_{1} l_{\mathrm{c} 3} \mathrm{c}_{23} \\
M_{31}=M_{13}=I_{3 \mathrm{zz}}+m_{3}\left(l_{\mathrm{c} 3}^{2}+l_{2} l_{\mathrm{c} 3} \mathrm{c}_{3}\right)+m_{3} l_{1} l_{\mathrm{c} 3} \mathrm{c}_{23} \\
M_{22}=I_{2 z z}+I_{3 z z}+m_{2} l_{\mathrm{c} 2}^{2}+m_{3}\left(l_{2}^{2}+l_{\mathrm{c} 3}^{2}+2 l_{2} l_{\mathrm{c} 3} \mathrm{c}_{3}\right) \\
M_{32}=M_{23}=I_{3 z z}+m_{3}\left(l_{\mathrm{c} 3}^{2}+l_{2} l_{\mathrm{c} 3} \mathrm{c}_{3}\right) \\
M_{33}=I_{3 z z}+m_{3} l_{\mathrm{c} 3}^{2}
\end{gathered}
$$

The vector of the centrifugal and Coriolis forces $\mathbf{C}(q, \dot{q})$ is shown in Eq. (14):

$$
\begin{gathered}
\mathbf{C}=\left[\begin{array}{lll}
C_{11} & C_{21} & C_{31}
\end{array}\right]^{\mathrm{T}} \\
C_{11}=-2 l_{1}\left(m_{2} l_{\mathrm{c} 2} \mathrm{~s}_{2}+m_{3} l_{2} \mathrm{~s}_{2}\right) \dot{\theta}_{1} \dot{\theta}_{2}-2 l_{1} m_{3} l_{\mathrm{c} 3} \mathrm{~s}_{23} \cdot \dot{\theta}_{1} \dot{\theta}_{2}-\ldots \\
\left(m_{2} l_{1} l_{\mathrm{c} 2} \mathrm{~s}_{2}+m_{3} l_{1}\left(l_{2} \mathrm{~s}_{2}+l_{\mathrm{c} 3} \mathrm{~s}_{23}\right)\right) \dot{\theta}_{2}^{2}-m_{3} l_{\mathrm{c} 3}\left(l_{2} \mathrm{~s}_{3}+l_{1} \mathrm{~s}_{23}\right) \dot{\theta}_{3}^{2}-\ldots \\
-2 l_{\mathrm{c} 3} m_{3}\left(l_{2} \mathrm{~s}_{3}+l_{1} \mathrm{~s}_{23}\right) \dot{\theta}_{1} \dot{\theta}_{3}-2 m_{3} l_{\mathrm{c} 3}\left(l_{2} \mathrm{~s}_{3}+l_{1} \mathrm{~s}_{23}\right) \dot{\theta}_{2} \dot{\theta}_{3} \\
C_{21}=m_{3}\left(l_{1} l_{2} \mathrm{~s}_{2}+l_{1} l_{\mathrm{c} 3} \mathrm{~s}_{23}\right) \dot{\theta}_{1}^{2}+m_{2} l_{1} l_{\mathrm{c} 2} \mathrm{~s}_{2} \cdot \dot{\theta}_{1}^{2}-\ldots \\
2 m_{3} l_{2} l_{\mathrm{c} 3} \mathrm{~s}_{3} \cdot \dot{\theta}_{1} \dot{\theta}_{3}-2 m_{3} l_{2} l_{\mathrm{c} 3} \mathrm{~s}_{3} \cdot \dot{\theta}_{2} \dot{\theta}_{3}-m_{3} l_{2} l_{\mathrm{c} 3} \mathrm{~s}_{3} \cdot \dot{\theta}_{3}^{2} \\
C_{31}=m_{3}\left(l_{2} l_{\mathrm{c} 3} \mathrm{~s}_{3}+l_{1} l_{\mathrm{c} 3} \mathrm{~s}_{23}\right) \dot{\theta}_{1}^{2}+m_{3} l_{2} l_{\mathrm{c} 3} \mathrm{~s}_{3}\left(2 \dot{\theta}_{1} \dot{\theta}_{2}+\dot{\theta}_{2}^{2}\right)
\end{gathered}
$$

The vector of the gravitational force $\mathbf{G}(q)$ is shown in Eq. (18) [17]. Since the motion of the 3-DOF manipulator is contained in the $x-y$ plane, the gravitational force is zero.

$$
\mathbf{G}=\left[\begin{array}{lll}
0 & 0 & 0
\end{array}\right]^{\mathrm{T}}
$$

\section{Desired Trajectory of the 3-DOF Manipulator}

To control the 3-DOF manipulator it is necessary to know at every instant of time the trajectory that it follows, i.e., its position with respect to the desired reference system.

From the kinematic and dynamic models of the robotized manipulator, obtained in the previous section, a trajectory is established in Cartesian space to generate, through inverse kinematics, the corresponding joint trajectories, which after making adequate interpolations, must be entered into the robot's control system. This control system, based on the manipulator's dynamic model, considers the control algorithms needed for the correct performance of the robot following the Cartesian trajectory, getting the curves corresponding to the different stages of the system.

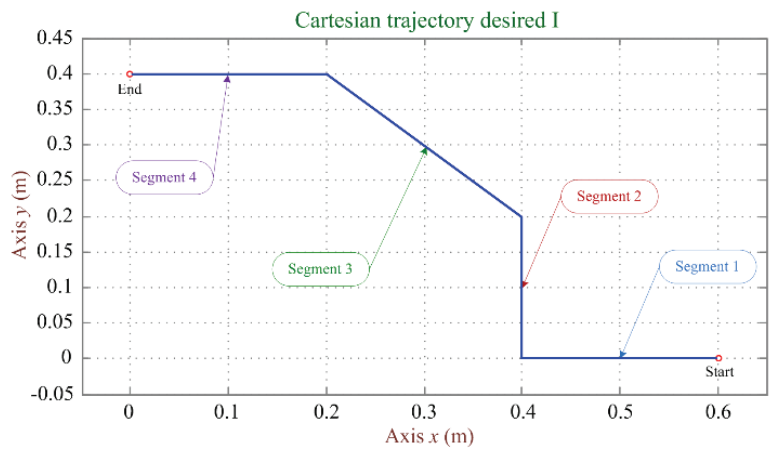

Fig. 2. Desired cartesian trajectory I for the 3-DOF manipulator

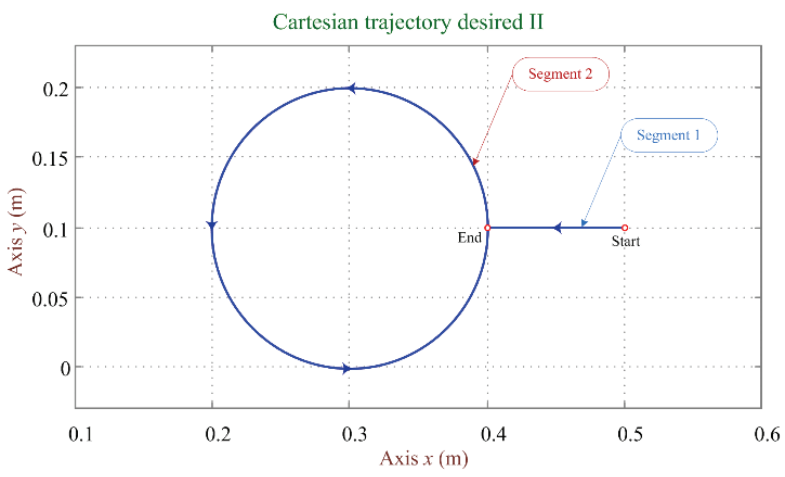

Fig. 3. Desired cartesian trajectory II for the 3-DOF manipulator

\subsection{Trajectories test}

The selection of the trajectory that must be entered into the control system to carry out the corresponding performance tests of the control algorithms considers the tracking requirements given by a combination of straightline trajectories composed of four segments as well as a circle path, as seen in Fig. 2 and in Fig. 3, respectively.

\section{Simulation Environment and real-time Execution}

The simulation and real-time execution will be based on five control laws: predictive; hyperbolic sine-cosine; sliding mode; hybrid composed of a predictive + hyperbolic sine-cosine controller; and adaptive controller. Fig. 4 is a schematic of the simulation environment of the

Table 1. Parameter values of the manipulator.

\begin{tabular}{|c|c|c|c|c|}
\hline Parameter & Link 1 & Link 2 & Link 3 & Units \\
\hline$l$ & 0.2 & 0.2 & 0.2 & {$[\mathrm{~m}]$} \\
$l_{c}$ & 0.0229 & 0.0229 & 0.0983 & {$[\mathrm{~m}]$} \\
$m$ & 2.0458 & 2.0458 & 3.6236 & {$[\mathrm{Kg}]$} \\
$I_{z z}$ & 0.0116 & 0.0116 & 0.0673 & {$\left[\mathrm{Kg} \cdot \mathrm{m}^{2}\right]$} \\
$F_{v}$ & 0.025 & 0.025 & 0.025 & {$[\mathrm{n} \cdot \mathrm{m} \cdot \mathrm{s} / \mathrm{rad}]$} \\
$F_{\text {eca }}$ & 0.05 & 0.05 & 0.05 & {$[\mathrm{~N} \cdot \mathrm{m}]$} \\
$F_{e c b}$ & 0.05 & 0.05 & 0.05 & {$[\mathrm{~N} \cdot \mathrm{m}]$} \\
\hline
\end{tabular}


Table 2. Parameter values of the actuator.

\begin{tabular}{|c|c|c|c|c|}
\hline Parameter & Servo 1 & Servo 2 & Servo 3 & Units \\
\hline$R_{a}$ & 1.6 & 1.6 & 1.6 & {$[\Omega]$} \\
$L_{a}$ & 0.0048 & 0.0048 & 0.0048 & {$[\mathrm{H}]$} \\
$J_{m}$ & 0.007 & 0.007 & 0.007 & {$\left[\mathrm{Kg} \cdot \mathrm{m}^{2}\right]$} \\
$B_{m}$ & 0.01313 & 0.01313 & 0.01313 & {$[\mathrm{~N} \cdot \mathrm{m} \cdot \mathrm{s} / \mathrm{rad}]$} \\
$K_{a}$ & 0.35 & 0.35 & 0.35 & {$[\mathrm{~N} \cdot \mathrm{m} / \mathrm{A}]$} \\
$K_{b}$ & 0.04 & 0.04 & 0.04 & {$[\mathrm{~V} \cdot \mathrm{s} / \mathrm{rad}]$} \\
$F_{\text {eca }}$ & 0.00625 & 0.00625 & 0.00625 & {$[\mathrm{~N} \cdot \mathrm{m}]$} \\
$F_{e c b}$ & 0.00625 & 0.00625 & 0.00625 & {$[\mathrm{~N} \cdot \mathrm{m}]$} \\
$n$ & $1 / 600$ & $1 / 561.6$ & $1 / 561.6$ & {$[$ Times $]$} \\
$A$ & 15 & 15 & 15 & {$[$ Times] } \\
$K_{s}$ & 10 & 10 & 10 & {$[$ Times] } \\
$K_{p}$ & 1 & 1 & 1 & {$[$ Times] } \\
$P$ & 1 & 1 & 1 & {$[$ Times] } \\
\hline
\end{tabular}

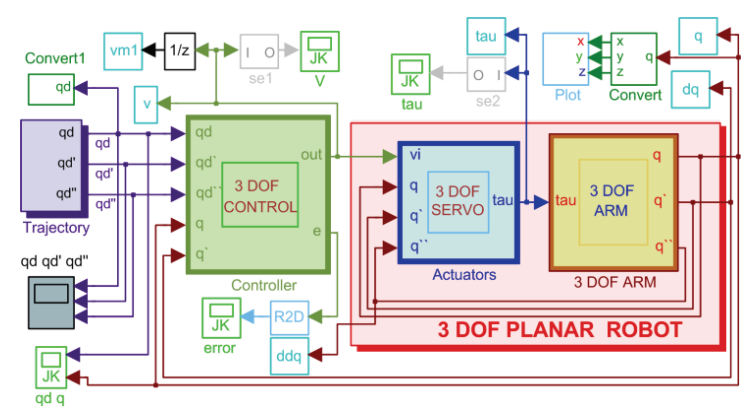

Fig. 4. Block diagram of the robot, actuators and controllers to be simulated

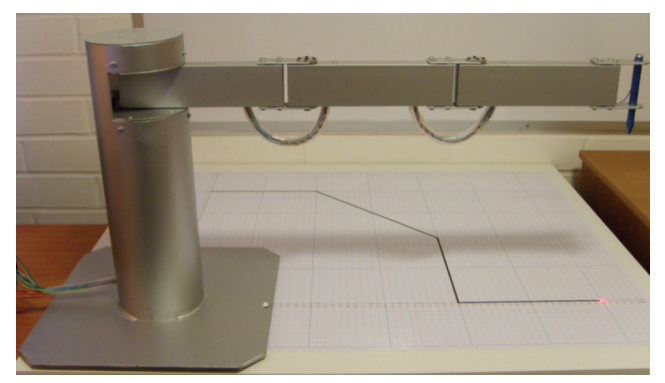

Fig. 5. Real redundant manipulator used to run the control laws: trajectory I

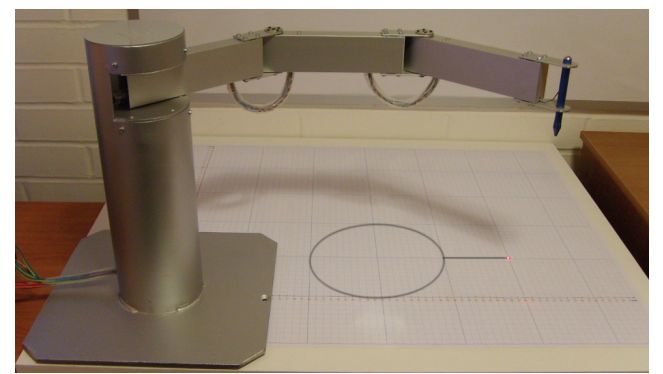

Fig. 6. Real redundant manipulator used to run the control laws: trajectory II

dynamic model of the 3-DOF manipulator, for which the programming tools of the MatLab/Simulink software are used. Table 1 shows the values of the parameters considered for the 3-DOF manipulator that is being studied.

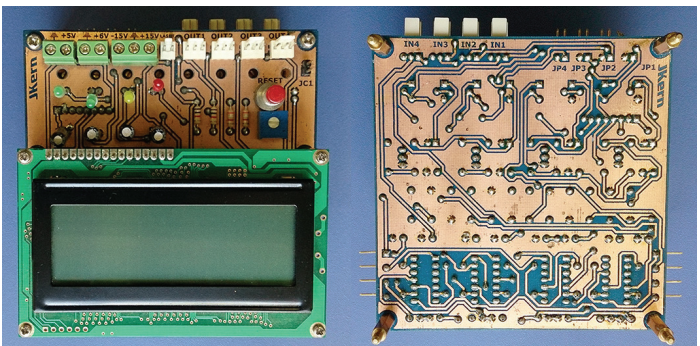

Fig. 7. Communication interface and signal conditioning

Table 2 presents the values of the parameters considered for each of the actuators of the 3-DOF manipulator that is being studied, corresponding to direct current servomotors.

Fig. 5 and Fig. 6 show the real redundant 3-DOF manipulator according to the I (combination of straightline) and II (combination of straight-line and circle path) test trajectories, respectively.

Fig. 7 shows the communication interface and signal conditioning used to run in real time the control algorithms on the real redundant 3-DOF manipulator.

\section{Results of the Controllers' Performance}

The simulation results obtained when the manipulator robot is made to follow the desired trajectories are described below. The trajectories are presented by means of Fig. 2 and Fig. 3, using each of the proposed controllers.

\subsection{Predictive controller}

The performance of the predictive control is based on the squared weighted sum of errors and control movements [13]. At each instant $t$ an optimum control problem is solved over a finite and future horizon $N$ where a function $f$ is minimized that quantifies the difference between the output of the plant $(y)$ and the reference $(r)$, and the control effort $(u)$ [14],

$$
\min _{u} f(|y-r|,|u|)
$$

subject to the following restrictions:

$$
y_{\min } \leq y \leq y_{\max }, u_{\min } \leq u \leq u_{\max }, \Delta u_{\min } \leq u \leq \Delta u_{\max }
$$

That is, at every instant $t$, from the model of the system a control sequence is calculated over a finite horizon $N$ from the current state $x(t)[15]$.

$$
u(t \mid t), u(t+1 \mid t), u(t+2 \mid t), \cdots u(t+N \mid t)
$$

\subsubsection{Behaviour of the predictive controller}

The configuration of the predictive controller parameters 
Table 3. Parameters for predictive controller.

\begin{tabular}{|c|c|c|}
\hline Parameter & Value & Units \\
\hline Control intervals & $6 \times 10^{-5}$ & [seconds] \\
Prediction horizon & 300 & [intervals] \\
Control horizon & 1 & [intervals] \\
\hline
\end{tabular}

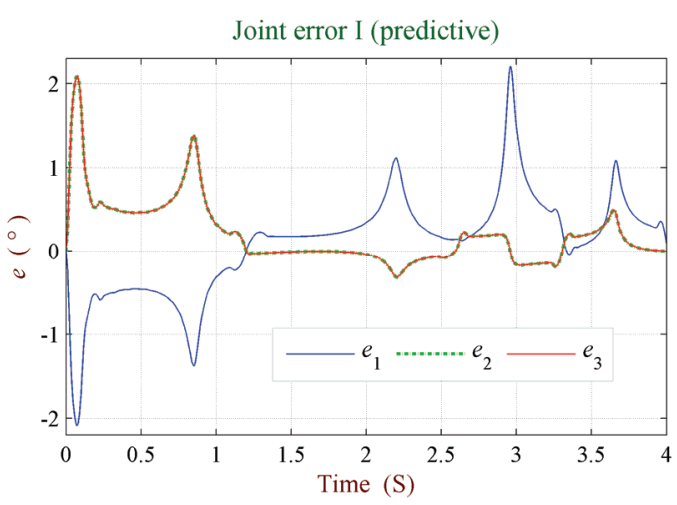

Fig. 8. Joint trajectory error using the predictive controller: trajectory I

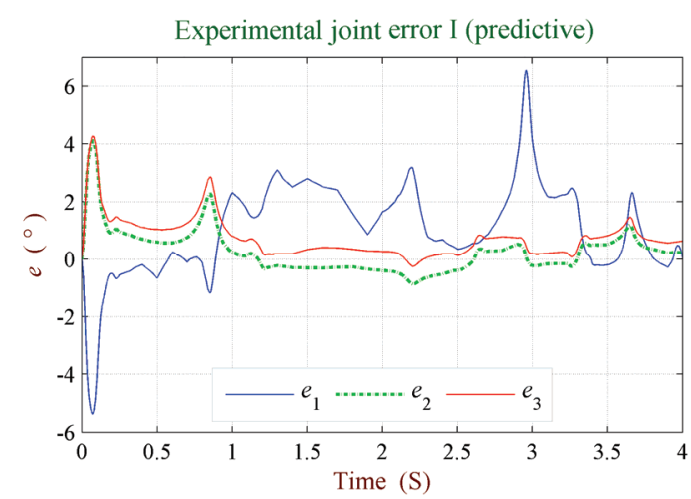

Fig. 9. Joint trajectory error using the predictive controller: trajectory I (experimental)

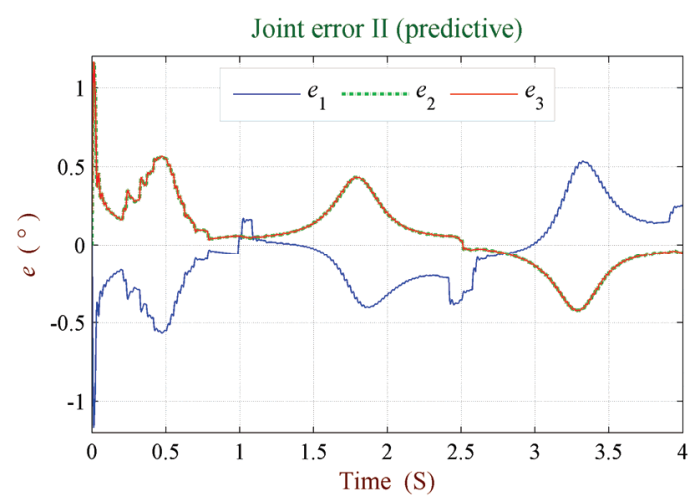

Fig. 10. Joint trajectory error using the predictive controller: trajectory II

are shown in Table 3.

Fig. 8 and Fig. 9 show the error curves obtained for the desired and real joint trajectories I: simulated and experimental, respectively, using the adaptive controller.

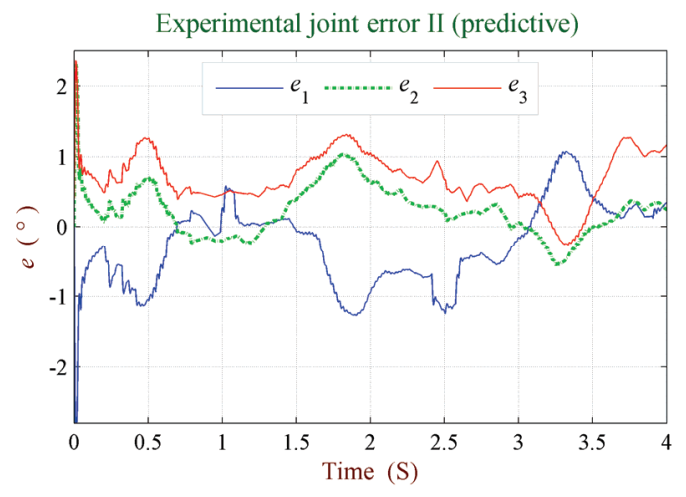

Fig. 11. Joint trajectory error using the predictive controller: trajectory II (experimental)

Fig. 10 and Fig. 11 show the error curves obtained for the desired and real joint trajectories II: simulated and experimental, respectively, using the adaptive controller.

\subsection{Hyperbolic sine-cosine controller}

The hyperbolic sine-cosine controller consists of a proportional part based on a hyperbolic sine and cosine, a derivative part based on a hyperbolic sine, and gravity compensation. The equations for this control algorithm are $(18-21)$ [17].

$$
\begin{gathered}
\boldsymbol{\tau}=\mathbf{K}_{\mathbf{p}} \sinh \left(\mathbf{q}_{\mathbf{e}}\right) \cosh \left(\mathbf{q}_{\mathbf{e}}\right)-\mathbf{K}_{\mathbf{v}} \sinh (\dot{\mathbf{q}})+\mathbf{G}(\mathbf{q}) \\
\mathbf{q}_{\mathbf{e}}=\mathbf{q}_{\mathbf{d}}-\mathbf{q} \\
\mathbf{K}_{\mathbf{p}}=\operatorname{diag}\left(K_{p 1}, K_{p 2} \ldots K_{p n}\right) \\
\mathbf{K}_{\mathbf{v}}=\operatorname{diag}\left(K_{v 1}, K_{v 2} \ldots K_{v n}\right)
\end{gathered}
$$

where, $\mathbf{K}_{\mathrm{p}}$ : Proportional gain, matrix, $\mathbf{K}_{\mathbf{v}}$ : Derivative gain matrix, $\mathbf{q}_{\mathbf{d}}$ : Position error vector of the joints, and $\mathbf{q}_{\mathbf{e}}$ : Desired position vector of the joints.

\subsubsection{Behavior of the hyperbolic sine-cosine controller}

Table 4 presents the values of the gains used in the hyperbolic sine-cosine controller of the three joints of the robot, according to Eqs. (20) and (21).

Table 4. Values of the gains for the hyperbolic sine-cosine controllers.

\begin{tabular}{c|cccccc}
\hline Gain & $K_{p 1}$ & $K_{p 2}$ & $K_{p 3}$ & $K_{v 1}$ & $K_{v 2}$ & $K_{v 3}$ \\
Value & 100 & 90 & 80 & 4 & 3.8 & 3.6 \\
\hline
\end{tabular}

Fig. 12 and Fig. 13 show the graphs of the errors obtained from the desired and real simulated, and desired and real experimental joint trajectories I, respectively, using the hyperbolic sine-cosine controller; $e_{n}$ express the errors in the joint trajectories ( $n$ represents joints 1 through 3).

Fig. 14 and Fig. 15 show the graphs of the errors 


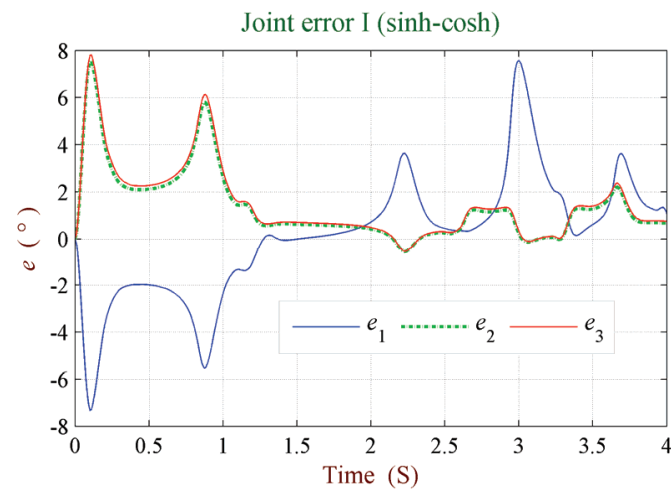

Fig. 12. Joint trajectory error using the hyperbolic sinecosine controller: trajectory I.

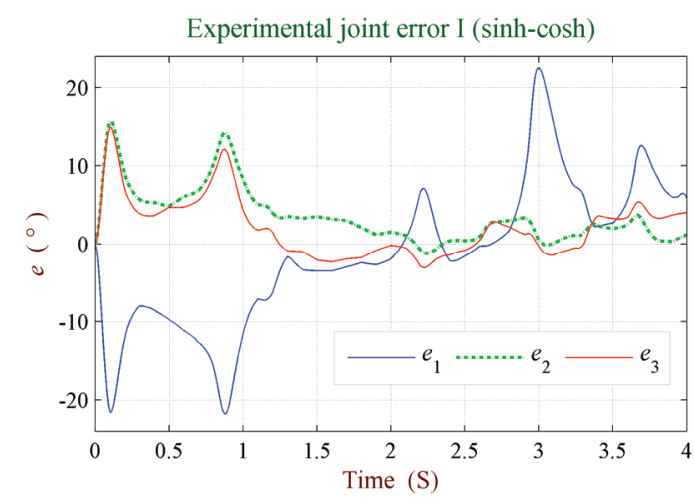

Fig. 13. Joint trajectory error using the hyperbolic sinecosine controller: trajectory I (experimental)

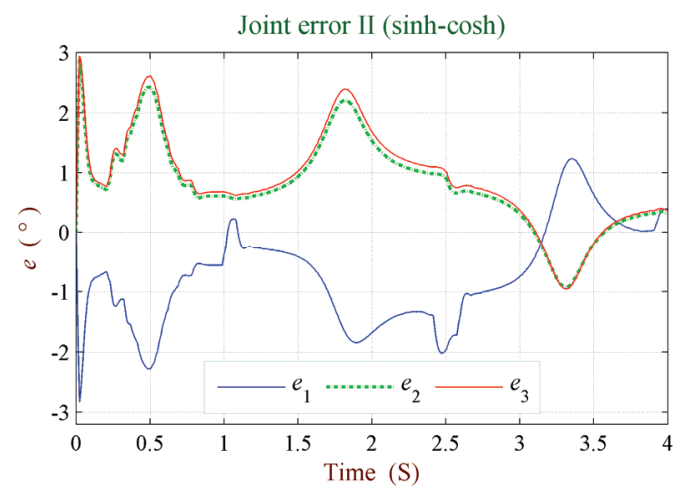

Fig. 14. Joint trajectory error using the hyperbolic sinecosine controller: trajectory II

obtained from the desired and real simulated, and desired and real experimental joint trajectories II, respectively, using the hyperbolic sine-cosine controller; $e_{n}$ express the errors in the joint trajectories ( $n$ represents joints 1 through 3).

\subsection{Predictive + hyperbolic sine-cosine controller}

To improve the response of the hyperbolic sine-cosine controller presented in section 6.2, the behaviour of the predictive controller is incorporated in an additive way.

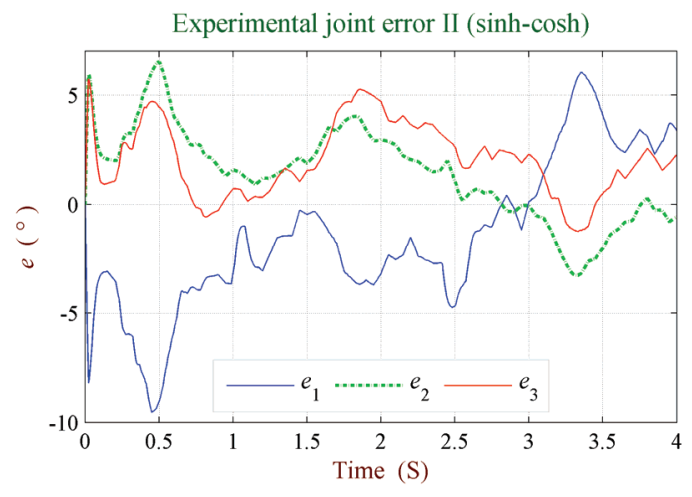

Fig. 15. Joint trajectory error using the hyperbolic sinecosine controller: trajectory II (experimental)

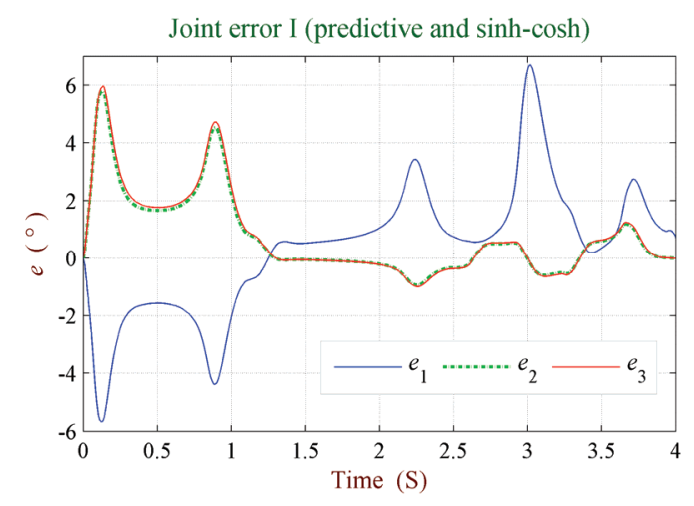

Fig. 16. Joint trajectory error using the predictive + sinhcosh controller: trajectory I

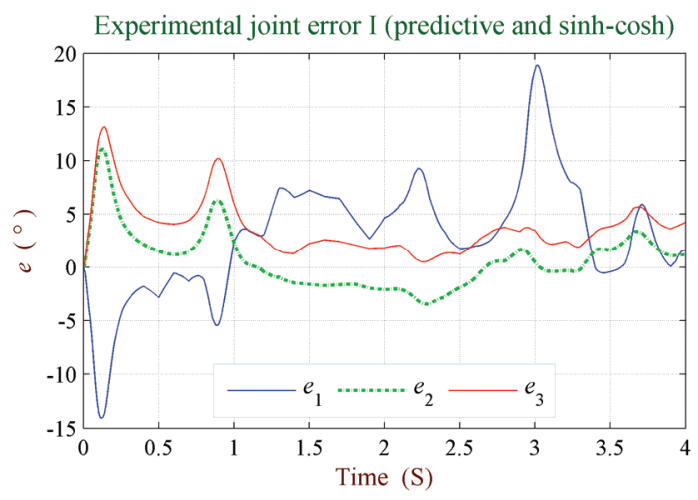

Fig. 17. Joint trajectory error using the predictive + sinhcosh controller: trajectory I (experimental)

\subsubsection{Behavior of the predictive + hyperbolic sine-cosine controller}

The gains of the predictive and sine-cosine controllers, which are used in the simulation and execution in real time of this controller, are shown in Table 3 and Table 4, respectively. Fig. 16 and Fig. 17 show the graphs of the errors obtained from the desired and real simulated, and desired and real experimental joint trajectories I, respectively, using the predictive + hyperbolic sine-cosine controller; $e_{n}$ express the errors in the joint trajectories $(n$ 


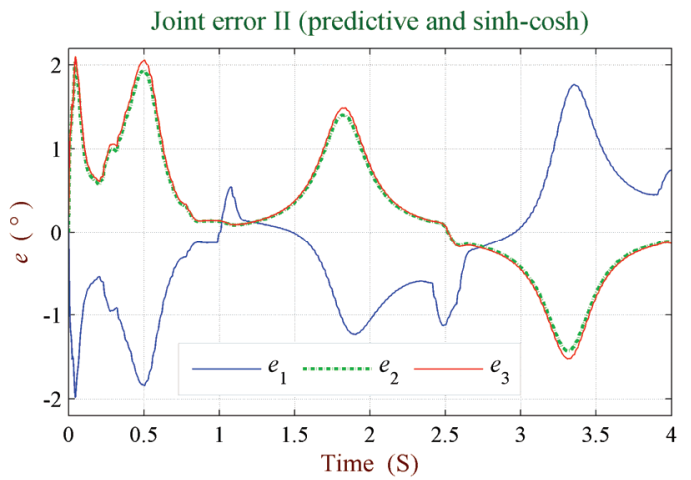

Fig. 18. Joint trajectory error using the predictive + sinhcosh controller: trajectory II.

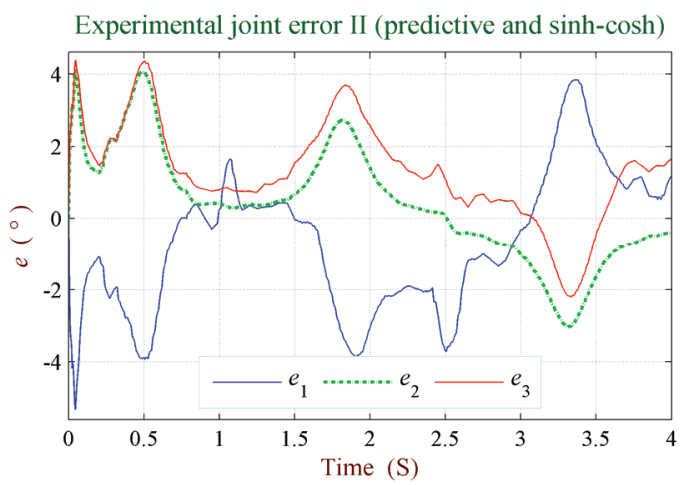

Fig. 19. Joint trajectory error using the predictive + sinhcosh controller: trajectory II (experimental)

represents joints 1 through 3 ).

Fig. 18 and Fig. 19 show the graphs of the errors obtained from the desired and real simulated, and desired and real experimental joint trajectories II, respectively, using the predictive + hyperbolic sine-cosine controller; $e_{n}$ express the errors in the joint trajectories ( $n$ represents joints 1 through 3 ).

\subsection{Sliding mode control}

The Sliding Mode Control (SMC) is a robust control strategy and particularly a variable structure control technique. It is based on the definition of a control law that commutes at high frequency and succeeds in taking the state of the system to a surface called the sliding surface, and once on it, keeps it from possible external perturbations.

The SMC techniques are known for being robust, having a direct design, and providing a solid solution for fault estimation, control and diagnosis [18-20].

The robustness of the SMC can be improved by shortening the time required to get the sliding mode, or it can be guaranteed during whole control action intervals, eliminating the reach phase. The control law of the SMC corresponds to [16] and is represented by:

$$
\boldsymbol{\tau}=-\mathbf{K} \cdot \operatorname{sgn}(\mathbf{s})
$$

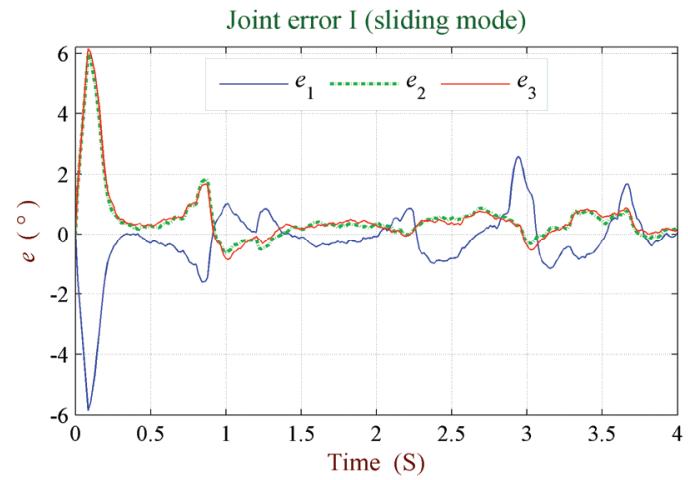

Fig. 20. Joint trajectory error using the sliding mode controller: trajectory I.

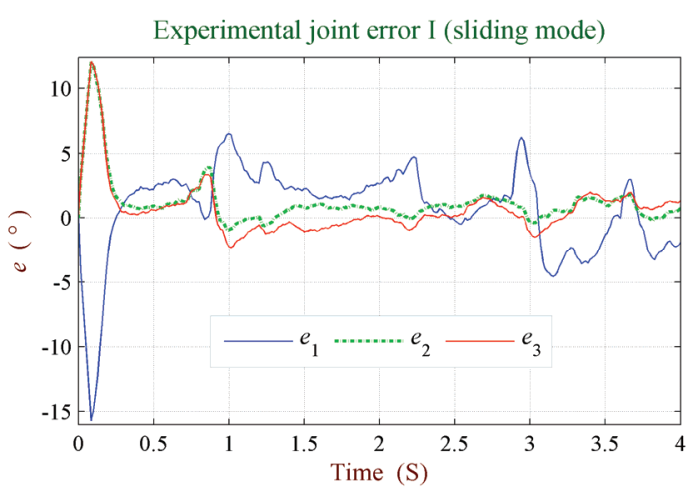

Fig. 21. Joint trajectory error using the sliding mode controller: trajectory I (experimental)

$$
\mathbf{K}=\operatorname{diag}\left(K_{1}, K_{2}, \ldots K_{n}\right)
$$

where, K: positive defined diagonal matrix (with dimension $n \times n)$, and the sliding surface is given by:

$$
\begin{gathered}
\mathbf{s}=\mathbf{W} \cdot\left(\mathbf{q}-\mathbf{q}_{\mathbf{d}}\right)+\left(\dot{\mathbf{q}}-\dot{\mathbf{q}}_{\mathbf{d}}\right) \\
\mathbf{W}=\operatorname{diag}\left(W_{1}, W_{2}, \ldots W_{n}\right)
\end{gathered}
$$

\subsubsection{Behavior of the sliding mode controller}

Table 5 shows the values of the gains used for the sliding mode controller according to Eqs. (23) and (25).

Table 5. Values of the gains for the sliding mode controller.

\begin{tabular}{c|c|c|c|c|c|c}
\hline Gain & $W_{1}$ & $W_{2}$ & $W_{3}$ & $K_{1}$ & $K_{2}$ & $K_{3}$ \\
\hline Value & 10 & 10 & 10 & 2.4 & 2.3 & 2.2 \\
\hline
\end{tabular}

Fig. 20 and Fig. 21 show the graphs of the errors obtained from the desired and real simulated, and desired and real experimental joint trajectories I, respectively, using the sliding mode controller; $e_{n}$ express the errors in the joint trajectories ( $n$ represents joints 1 through 3 ).

Fig. 22 and Fig. 23 show the graphs of the errors obtained from the desired and real simulated, and desired and real experimental joint trajectories II, respectively, 


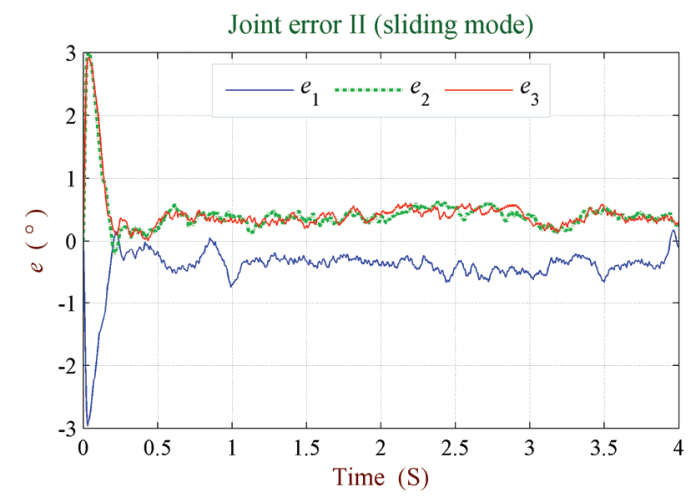

Fig. 22. Joint trajectory error using the sliding mode controller: trajectory II

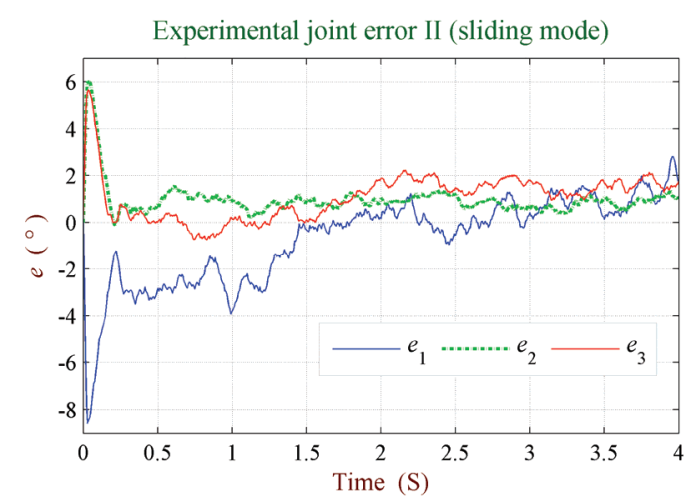

Fig. 23. Joint trajectory error using the sliding mode controller: trajectory II (experimental)

using the sliding mode controller; $e_{n}$ express the errors in the joint trajectories ( $n$ represents joints 1 through 3 ).

\subsection{Adaptive controller}

Adaptive Control is a control whose parameters vary constantly due to changes in the plant (manipulator robot) or to external perturbations [21].

The adaptive control is constituted by the online estimation of the plant parameters obtained by processing the input, $u$, and output, $y$, signals the calculation of the controller parameters $(\theta(t))$ based on the estimated plant parameters, and a control law whose parameters are $\theta(\mathrm{t})$.

\subsubsection{Behavior of the adaptive controller}

Table 6 shows the gain values used for the adaptive controller.

Table 6. Gain values for the adaptive controller.

\begin{tabular}{|c|c|c|c|c|c|c|c|c|c|}
\hline Gain & $\overline{L_{1}}$ & $L_{2}$ & $L_{3}$ & $G_{a 1}$ & $G_{a 2}$ & $G_{a 3}$ & $K_{v 1}$ & $K_{v 2}$ & $K_{v 3}$ \\
\hline Value & 8 & 8 & 8 & 0.1 & 0.1 & 0.1 & 20 & 20 & 20 \\
\hline
\end{tabular}

Fig. 24 and Fig. 25 show the error curves obtained for the desired and real joint trajectories I: simulated and

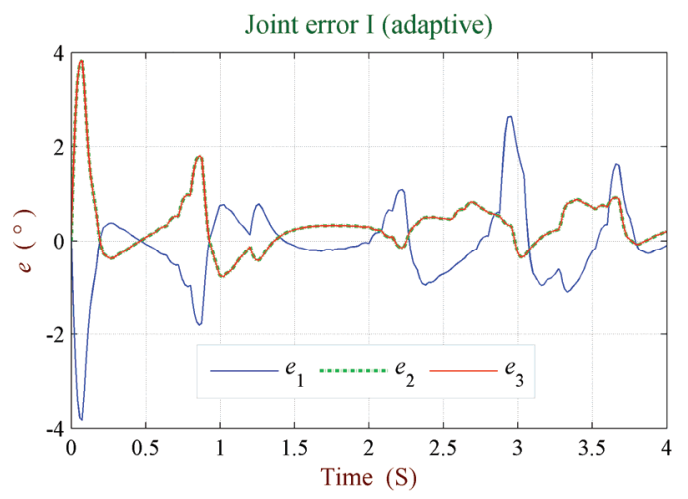

Fig. 24. Joint trajectory error using the adaptive controller: trajectory I

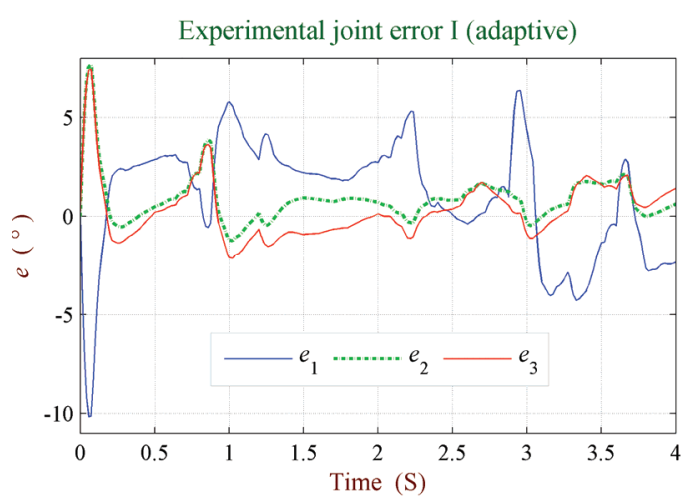

Fig. 25. Joint trajectory error using the adaptive controller: trajectory I (experimental)

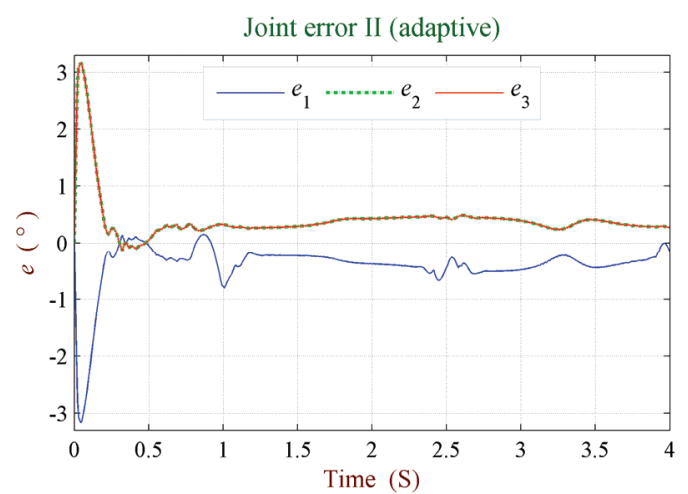

Fig. 26. Joint trajectory error using the adaptive controller: trajectory II

experimental, respectively, using the adaptive controller.

Fig. 26 and Fig. 27 show the error curves obtained for the desired and real joint trajectories II: simulated and experimental, respectively, using the adaptive controller.

\section{Synthesis of Results and Performance Analysis}

A comparison is now made of the manipulator robot's dynamic performance obtained during the motion of each 


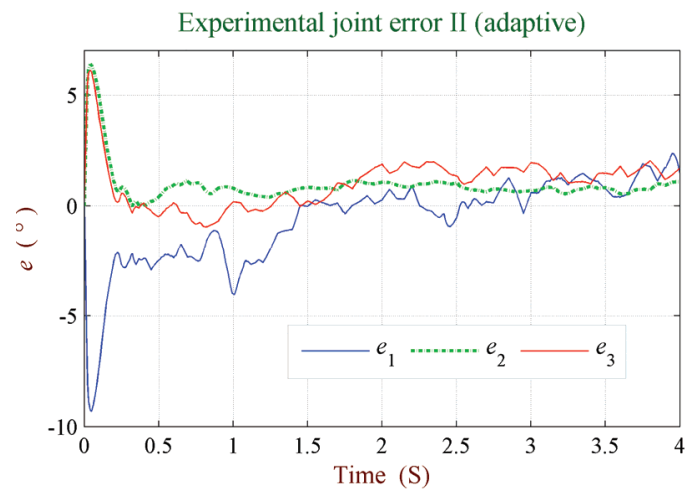

Fig. 27. Joint trajectory error using the adaptive controller: trajectory II (experimental)

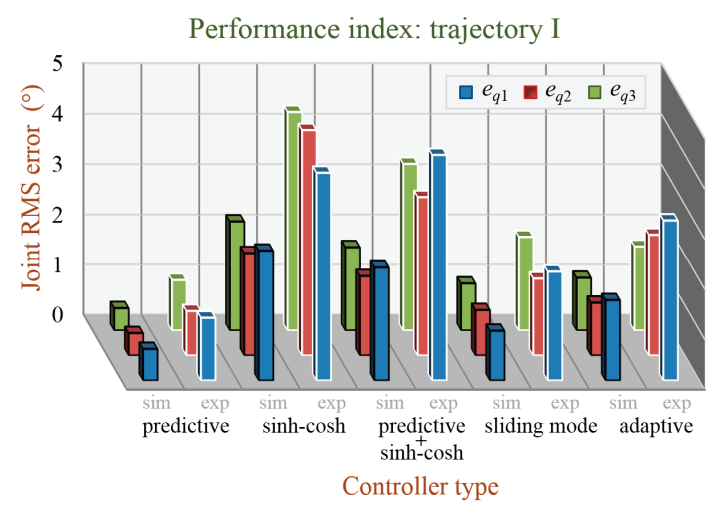

Fig. 28. Performance index corresponding to the joint's trajectory I (simulated and experimental)

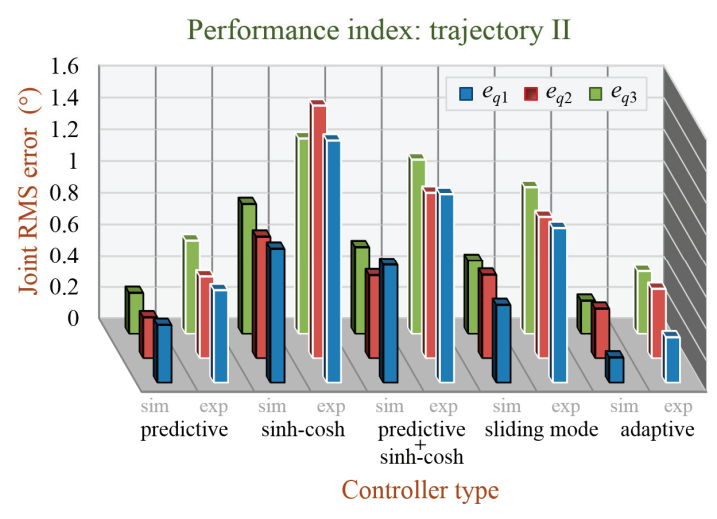

Fig. 29. Performance index corresponding to the joint's trajectory II (simulated and experimental)

of its three joints when following the imposed trajectories, which were presented in Fig. 2 and Fig. 3. This is done considering the RMS joint error indices defined by (26):

$$
\mathrm{RMS}=\sqrt{\frac{1}{n} \sum_{i=1}^{n} e_{i}^{2}}
$$

where $e_{i}$ : trajectory errors and $\mathrm{n}$ : number of data.

In this way it is also possible to make a comparison of the performance of all the controllers used considering joint trajectory errors. The results of the performance of each controller are shown graphically by means of Fig. 28 and Fig. 29, where it is seen that the best results were given by the predictive controller, according to the criteria corresponding to each RMS joint error index.

\section{Conclusions and Future Development}

Controlling the motion of a manipulator robot's joints is a current issue whose study is of great importance in order to make its terminal effectors follow a predefined trajectory with minimum deviations. The nonlinear behavior and the high performance requirement are the main problems that appear in the design of manipulator robots and their controllers. For that reason, the design, simulation and comparison of the performance of controllers applied to a robot with three Degrees of Freedom were presented. Five controllers with their corresponding control laws were developed to test the robot's dynamic model: predictive; hyperbolic sine-cosine; sliding mode; hybrid composed of a predictive + hyperbolic sine-cosine controller; and adaptive controller.

The kinematic and dynamic models of the manipulator with three degrees of freedom were described using the Denavit-Hartenberg and the Euler-Lagrange methods, respectively.

A simulator was developed by means of the MatLab/ Simulink software, which allows analysing the dynamic performance of the robot and of the designed controllers.

The manipulator robot was made to follow a step-type test trajectory. The results of the performance of this manipulator and of its controllers for each of the three joints were compared by means of RMS indices, considering joint errors according to the imposed trajectory and to the controller used.

The controller that gave the smallest RMS error between the desired and the real trajectory at each of the three joints was the predictive controller. However, it must be kept in mind that as the prediction horizon and the control intervals are increased, the simulation time also increases due to the different calculations that must be made for each interval within the prediction horizon.

In view of the results, future work considers using this predictive controller in manipulator robots with more degrees of freedom.

\section{Acknowledgments}

This work was supported by: 1. Proyectos Basales and the Vicerrectoría de Investigación, Desarrollo e Innovación of the Universidad de Santiago de Chile, Chile. 2. Escuela de Electrónica, Universidad del Azuay, Cuenca, Ecuador. 


\section{References}

[1] M. Ebad and B. M. Song, "Accurate Model Predictive Control of Bidirectional DC-DC Converters for DC Distributed Power Systems," Power and Energy Society General Meeting IEEE, pp. 1-8, 2012.

[2] J. Mu and D. Rees, "Approximate Model Predictive Control for Gas Turbine Engines," American Control Conference IEEE, vol. 6, pp. 5704-5709, 2004.

[3] M. Farrokhsiar and H. Najjaran, "Unscented Model Predictive Control of Chance Constrained Nonlinear Systems," Advanced Robotics, vol. 28, no. 4, pp. 257267, 2014

[4] E. Camacho and C. Bordons, "Control Predictivo: Pasado, Presente y Futuro," Revista Iberoamericana de Automática e Informática Industrial, vol. 1, no. 3, pp. 5-28, 2004.

[5] G. I. Suárez, O. A. Ortiz, P. M. Aballay and N. H. Aros, "Adaptive Neural Model Predictive Control for the Grape Juice Concentration Process," Industrial Technology. IEEE, pp. 57-63, 2010.

[6] M. Kinnaert, "Adaptive Generalized Predictive Controller for MIMO Systems," International Journal of Control, vol. 50, no. 1, pp. 161-172, 1989.

[7] A. Dutta, R. De Keyser and I. Nopens, "Robust Nonlinear Extended Prediction Self-Adaptive Control (NEPSAC) of Continuous Bioreactors," Control \& Automation IEEE, pp. 658-664, 2012.

[8] M. Moradi and H. Malekizade, "Neural Network Identification Based Multivariable Feedback Linearization Robust Control for a Two-Link Manipulator," Journal of Intelligent \& Robotic Systems, vol. 72, no. 2, pp. 167-178, Nov.2013.

[9] P. Boscariol, A. Gasparetto, and V. Zanotto, "Model Predictive Control of a Flexible Links Mechanism," Journal of Intelligent and Robotic Systems, vol. 58, no. 2, pp. 125-147, 2010.

[10] D. M. Anand, T. Selvaraj and S. Kumanan, "Fault Detection and Isolation in Robotic Manipulator Via Hybrid Neural Networks," International Journal of Simulation Modelling, vol. 7, no. 1, pp. 5-16, 2008.

[11] L. Acosta, S. Torres, J. Méndez, N. Marichal, A. Hamilton and L. Moreno, "Control Predictivo Mediante Interpolación Aplicado a un Robot Manipulador de Dos Articulaciones," Grupo de Computadoras y Control, Departamento de Física Fundamental y Experimenta, 2000.

[12] K. Prema, N. Senthil Kumar and Subhransu Sekhar Dash, "Online Control of DC Motors Using Fuzzy Logic Controller for Remote Operated Robots," Journal Electrical Engineering \& Technology, vol. 9, no. 1, pp. 352-362, 2014.

[13] M. Rau and D. Schroder, "Model Predictive Control with Nonlinear State Space Models," Advanced Motion Control IEEE, pp. 136-141, 2002.

[14] L. Orihuela, T. Álamo, D. Muñoz and F. Rubio,
“Algoritmo de Minimización para Control Predictivo con Restricciones," XXIX Jornadas de Automática, Departamento de Ingeniería de Sistemas y Automática, Universidad de Sevilla, Tarragona, Spain, 2008.

[15] M. De la Fuente and T. Calonge, "Aplicaciones de las Redes de Neuronas en Supervisión," Diagnosis y Control de Procesos. Editorial Texto, Equinoccio, Venezuela, 1999.

[16] Kern, J. "Análisis e Implementación de Sistemas de Control Tolerantes a Fallas Aplicados en un Robot de Tipo Manipulador Industrial Real". Master Thesis. Universidad de Santiago de Chile, Sep. 2010.

[17] C. Urrea and J. Kern, "Modeling, Simulation and Control of a Redundant SCARA-Type Manipulator Robot," International Journal of Advanced Robotic Systems, vol. 9, no. 58, pp. 01-14, 2012.

[18] A. Boucheta, I. K. Bousserhane, A. Hazzab, P. Sicard and M. K. Fellah, "Speed Control of Linear Induction Motor using Sliding Mode Controller Considering the End Effects," Journal of Electrical Engineering \& Technology vol. 7, no. 1, pp. 34-45, 2012.

[19] K. Braikia, M. Chettouh, B. Tondu, P. Acco, and M. Hamerlain, "Improved Control Strategy of 2-Sliding Controls Applied to a Flexible Robot Arm," Advanced Robotics, vol. 25, no. 11-12, pp. 1515-1538, 2011.

[20] G. Bartolini, A. Ferrara and E. Usani, "Chattering Avoidance by Second-Order Sliding Mode Control," Automatic control, IEEE, vol. 43, no. 2, pp. 241-246, 1998.

[21] J. Vojtesek and P. Dostál, "Simulation of Adaptive Control of Continuous Stirred Tank Reactor," International Journal of Simulation Modelling, vol. 8, no. 3, 133-144, 2009.

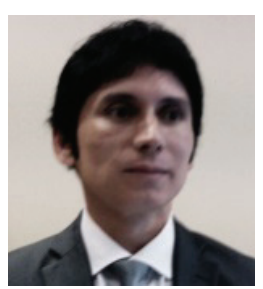

John Kern He received the M.Sc. Eng. and the Dr. degrees from Universidad de Santiago de Chile, Santiago, Chile in 2010 and 2013, respectively. John Kern is currently Professor of electronic engineering in the areas of automatic control and robotics. He has developed laboratories: theory and control systems, signals and communication system, and electronics.

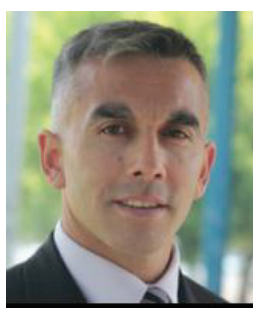

Claudio Urrea He received the M.Sc. Eng. and the Dr. degrees from Universidad de Santiago de Chile, Santiago, Chile in 1999, and 2003, respectively; and the Ph.D. degree from Institut National Polytechnique de Grenoble, France in 2003. Ph.D. Urrea is currently Professor at the Department 
of Electrical Engineering, Universidad de Santiago de Chile, from 1998. He has developed and implemented a Robotics Laboratory, where intelligent robotic systems are development and investigated. $\mathrm{He}$ is currently Director of the Doctorate in Engineering Sciences, Major in Automation, at the Universidad de Santiago de Chile.

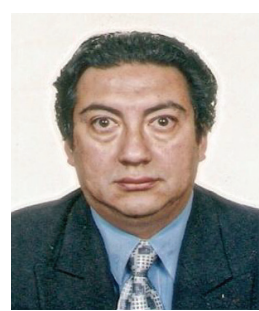

Hugo Torres He was born in Cuenca, Ecuador. He received the Electronic Engineering degree from Universidad Politécnica Salesiana, and Doctor of Research and Planning degree from Universidad Técnica de Loja. He received the M.Sc. Eng. and the Dr. degrees from Universidad de Santiago de Chile, Santiago, Chile in 2014. Hugo Torres is currently Professor at the Universidad del Azuay from 1991, automatic member of the Universidad de Santiago de Chile. He has developed and implemented industrial control laboratories, PLC and electronics. 